\title{
Evaluation of patient organ doses from kilovoltage cone-beam CT imaging in radiation therapy
}

\author{
Alper Özseven ${ }^{1}$, Bahar Dirican $\oplus^{2}$ \\ ${ }^{1}$ Suleyman Demirel University, Medical Faculty, Isparta, Turkey \\ ${ }^{2}$ University of Health Sciences, Gulhane Medical Faculty, Ankara, Turkey
}

\begin{abstract}
Background: Currently, CBCT system is an indispensable component of radiation therapy units. Because of that, it is important in treatment planning and diagnosis. CBCT is also an crucial tool for patient positioning and verification in image-guided radiation therapy (IGRT). Therefore, it is critical to investigate the patient organ doses arising from CBCT imaging. The purpose of this study is to evaluate patient organ doses and effective dose to patients from three different protocols of Elekta Synergy $\mathrm{XVI}$ system for KV CBCT imaging examinations in image guided radiation therapy.

Materials and methods: Organ dose measurements were done with thermoluminescent dosimeters in Alderson RANDO male phantom for head \& neck (H\&N), chest and pelvis protocols of the Elekta Synergy XVI kV CBCT system. From the measured organ dose, effective dose to patients were calculated according to the International Commission on Radiological Protection 103 report recommendations.

Results: For H\&N, chest and pelvis scans, the organ doses were in the range of $0.03-3.43 \mathrm{mGy}, 6.04-22.94 \mathrm{mGy}$ and $2.5-25.28$ $\mathrm{mGy}$, respectively. The calculated effective doses were $0.25 \mathrm{mSv}, 5.56 \mathrm{mSv}$ and $4.72 \mathrm{mSv}$, respectively.

Conclusion: The obtained results were consistent with the most published studies in the literature. Although the doses to patient organs from the $\mathrm{KVCBCT}$ system were relatively low when compared with the prescribed treatment dose, the amount of delivered dose should be monitored and recorded carefully in order to avoid secondary cancer risk, especially in pediatric examinations.
\end{abstract}

Key words: KV CBCT; equivalent organ dose; effective dose; image guided radiation therapy; Alderson Rando phantom Rep Pract Oncol Radiother 2021;26(2):251-258

\section{Introduction}

Cancer is still accepted as the deadliest among all diseases [1]. In order to cure cancer, several treatment modalities have been used in recent years. Radiation therapy is one of the main treatment methods used in cancer treatment along with surgery and chemotherapy. Especially, when the surgery and chemotherapy are not feasible, radiation therapy is the only treatment choice for some cancer forms. Additionally, due to the vital status of radiation therapy in many cancer types, treatment delivery accuracy of radiation therapy becomes critical for cancer patients [2]. One of the most important factors that make cancer treatment efficacious is irradiation of the targeted tumor tissue under appropriate conditions, as well as protecting normal tissues surrounding the tumor as much as possible. It is essential that the patient is positioned correctly before and during the treatment in order

Address for correspondence: Alper Özseven, Department of Radiation Oncology, Suleyman Demirel University Hospital, Isparta, Turkey, tel: (+90) 246211 9538; e-mail: alperozseven@sdu.edu.tr 
to get favorable results [3]. The first step of treatment delivery is correct positioning of the patient on a treatment couch. Although the technician can align the patient on the treatment couch correctly from the external perspective; the position of normal tissues or tumor may not be as they should be from the internal point of view. For this reason, imaging the corresponding part of the treatment region before treatment is prerequisite for perfect treatment delivery.

Nowadays, image guided radiation therapy (IGRT) is accepted to be the standard treatment modality especially for challenging treatment cases and, especially, for arc therapy. Generally, Cone Beam Computed Tomography (CBCT) is believed to be one of the most useful imaging methods in radiation therapy. Moreover, CBCT is not only used in cancer treatment for imaging head\& neck $(\mathrm{H} \& \mathrm{~N})$, thorax and pelvis regions, but also used in the examination of the mandible and nasal region in dentistry. Commonly, CBCT imaging systems are named in terms of the energies produced by the X-ray generator during imaging processes. The platforms which use million-voltage (MV) X-ray energies for imaging are called MV-CBCT. On the other hand, some of the systems use kilo-voltage $(\mathrm{kV}) \mathrm{X}$-ray energies for this process and these platforms are named kilo-voltage CBCT ( $\mathrm{kV}$-CBCT). They consist of a $\mathrm{kV}$ generator that produces $\mathrm{X}$-rays in kilo-volt energies and a flat-panel detector, most often called electronic portal imaging device (EPID), that detects these attenuated X-rays. During the imaging process the $\mathrm{kV}$-generator and flat-panel detector complete their rotation around the corresponding region of the body by obtaining high quality projection data [3-5].

Verifying position accuracy before and during the treatment of the patient is done by analyzing simultaneous images taken of the patient with the help of electronic portal imaging devices. EPID is a part of digital radiography that provides ease of use in imaging technology and equipped with the latest technological developments. In addition to AP and LAT images that are used conventionally at 90 degrees angle to each other, it is important to obtain a 3-dimensional image in order to establish patient position accuracy in all axes by using EPID [6]. On the other hand, beside having many advantages, the main disadvantage of this imaging system is delivering undesired radiation dose to the patient. The repeated use of a CBCT system in all fractions of treatment could increase the probability of developing secondary cancer risk, especially in pediatric patients $[7,8]$. Because of that, these imaging systems should be used carefully and the additional undesired imaging dose to patient organs should be monitored and measured.

The purpose of this study is to evaluate patient organ doses from three different protocols of the Elekta Synergy XVI system for kV CBCT imaging examinations in image guided radiation therapy using Alderson Rando male phantom. In addition to that, from the measured organ doses, effective dose to patients were calculated by using ICRP 103 recommendations.

\section{Materials and methods}

The measurements of patient organ doses from $\mathrm{kV}$-CBCT scans were collected by using Elekta Synergy Platform that was equipped with X-ray Volume Imaging (XVI) system release 4.5 (Elekta Oncology Systems Ltd, Crawley, UK) (Fig. 1). The X-ray source is attached to a retractable arm with a fixed source to isocenter distance of $100 \mathrm{~cm}$ (Fig. 1).

\section{Imaging process and measurements}

For H\&N, thoracic and pelvic scans; Head \& Neck S20, Chest M20 and Pelvis M20 presets were performed, respectively. The field of view in axial and longitudinal axis were described in detail in the study of Hyer et al. [9]. Moreover, the detailed

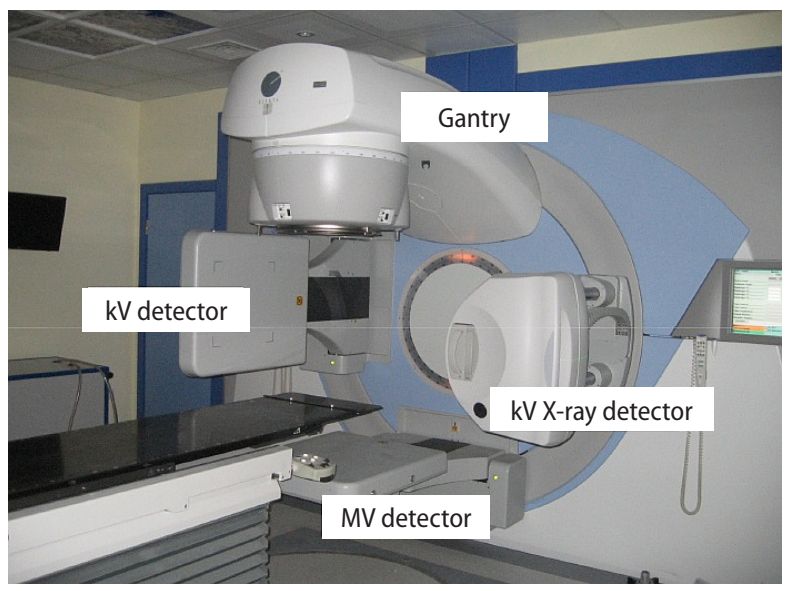

Figure 1. Elekta Synergy Platform equipped with XVI system 
Table 1. Exposure parameters for kV CBCT scanning protocols

\begin{tabular}{|l|c|c|c|} 
& Head \& Neck S20 & Chest M20 & Pelvis M20 \\
\hline X-ray voltage $[\mathrm{kVp}]$ & 100 & 120 & 120 \\
\hline X-ray $[\mathrm{mAs}]$ & 36.6 & 1056 & 360 \\
\hline Gantry rotation [degree] & 360 & 360 & 660 \\
\hline Nominal projection number & 366 & 660 & \\
\hline
\end{tabular}

parameters for each imaging protocol are listed in Table 1.

In this study a total of 20 patient organ dose measurements were carried out in an Alderson Rando anthropomorphic male phantom (Phantom Laboratory, Salem, NY, USA). In addition to that, effective doses to the patient were calculated from the measured organ doses. The detailed list of organs measured in the study is displayed in Table 2. In the Alderson Rando phantom, soft tissue, bone and lungs are equivalent to their true density. The bones in the Rando phantom are real human bones, and there are cavities equivalent to those in human body. The male RANDO phantom is made of 36 slices, each with a thickness of $2.5 \mathrm{~cm}$, except for slice 35 which is $9 \mathrm{~cm}$ thick. Each slice from slice number 1 to 34 contains a grid of holes, $5 \mathrm{~mm}$ in diameter, separated at $3 \mathrm{~cm}$ distance (Fig. 2). The positions of TLDs in the RANDO phantom were positioned in consistence with the recommendations of Golikov\&Nikitin and Scalzetti $[10,11]$.

The measurements were repeated three times for each scanning protocol. The arithmetic mean of these measurements was recorded as the average dose for the corresponding organ. The mean dose and standard deviation for each measurement were calculated by using the SPSS software version 22 (Armonk, NY, IBM Corp., USA).

Table 2. The measured organ doses (mGy) and calculated effective dose to patient (mSv) from single kV CBCT scan

\begin{tabular}{|c|c|c|c|}
\hline Organ & Head \& neck scan & Chest scan & Pelvic scan \\
\hline Brain & $2.80 \pm 0.01$ & & \\
\hline Oral mucosa & $3.25 \pm 0.01$ & & \\
\hline Salivary gland & $3.18 \pm 0.02$ & & \\
\hline Thyroid & $3.23 \pm 0.01$ & & \\
\hline Lens & $3.43 \pm 0.03$ & & \\
\hline Esophagus & $0.73 \pm 0.02$ & $15.04 \pm 0.08$ & \\
\hline Lungs & $0.03 \pm 0.00^{* *}$ & $17.83 \pm 0.08$ & \\
\hline Thymus & & $17.93 \pm 0.09$ & \\
\hline Spleen & & $15.26 \pm 0.08$ & \\
\hline Heart & & $22.94 \pm 0.10$ & \\
\hline Adrenals & & $11.93 \pm 0.06$ & \\
\hline Skin & $0.29 \pm 0.00 * *$ & $8.22 \pm 0.04$ & $2.89 \pm 0.02$ \\
\hline Liver & & $15.77 \pm 0.07$ & $2.50 \pm 0.02$ \\
\hline Stomach & & $11.70 \pm 0.06$ & $5.18 \pm 0.03$ \\
\hline Kidneys & & $6.04 \pm 0.03$ & $13.10 \pm 0.05$ \\
\hline Pancreas & & $7.88 \pm 0.04$ & $7.43 \pm 0.04$ \\
\hline Gall bladder & & & $23.52 \pm 0.09$ \\
\hline Small intestine & & & $25.28 \pm 0.09$ \\
\hline Colon & & & $24.25 \pm 0.09$ \\
\hline Bladder & & & $11.53 \pm 0.07$ \\
\hline Effective dose & $0.25^{*}$ & $5.56^{*}$ & $4.72^{*}$ \\
\hline
\end{tabular}

*Denotes underestimated effective doses for each scan (some of the organs with tissue factor couldn't be measured); ${ }^{* *}$ Denotes the standard deviation is less than 0.01 


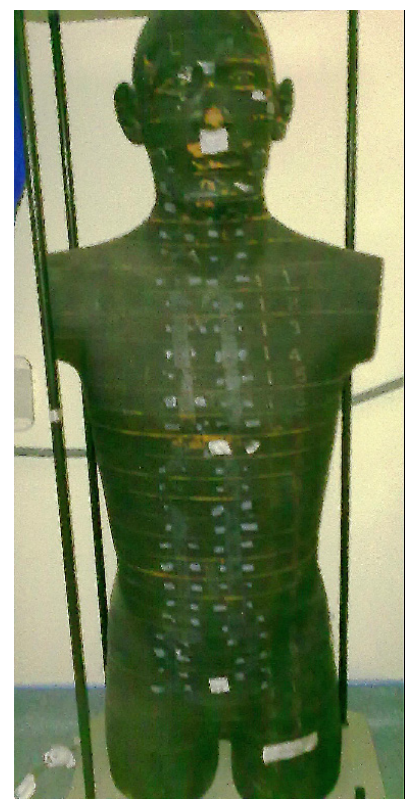

Figure 2. Alderson Rando male phantom

Scalzetti et al. offered a new proposal about the hole position of organs in the Alderson Rando phantom based on three-dimensional Cartesian coordinate system. According to that conjecture, the center hole of each slice was accepted as the $(0,0)$ central point in a rectangular frame (Fig. 3).

Lens and skin doses were measured by placing TLD on the surface of corresponding part of the phantom. Eight TLDs were used to compute the skin doses in each preset by locating the TLDs in the anterior, posterior, left and right surface of the phantom.

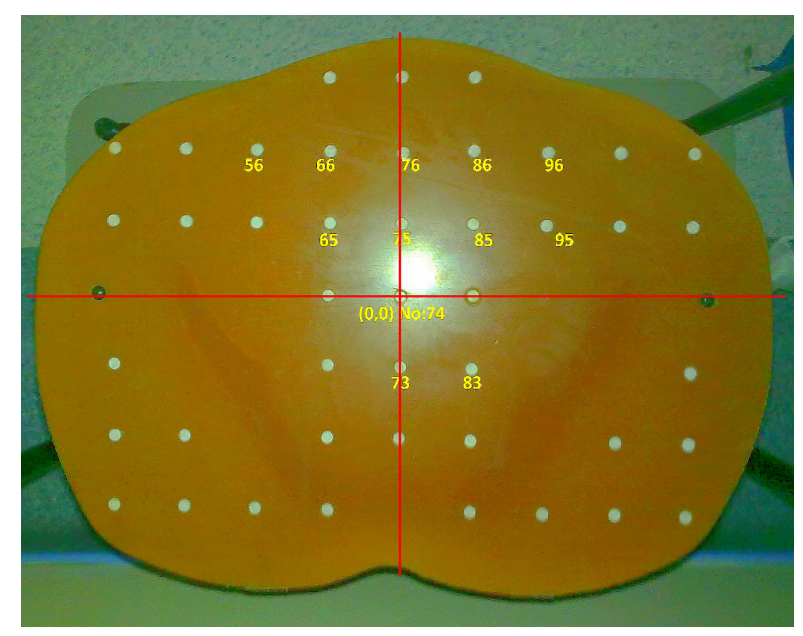

Figure 3. Center hole in Cartesian coordinate system assigned by Scalzetti et al. and hole numbers logic in Alderson Rando phantom (e.g. Slice 31)

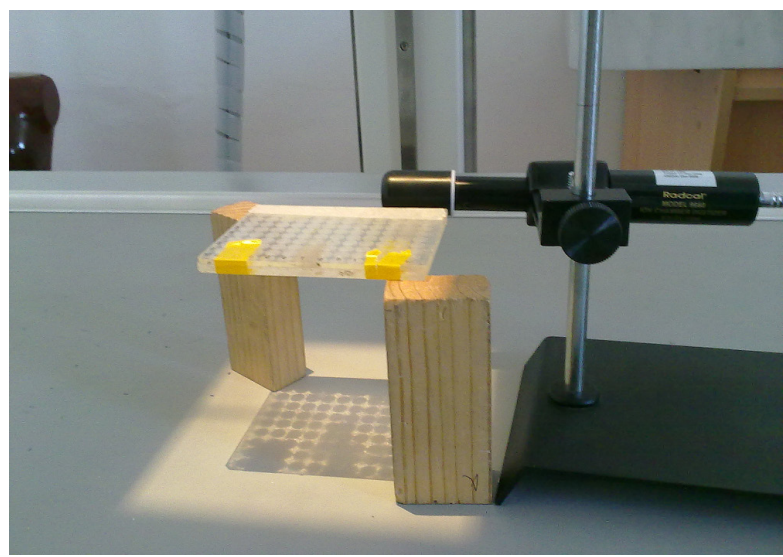

Figure 4. Calibration process of LiF TLDs

\section{TLD calibration}

Before starting the calibration process, Element Correction Coefficient (ECC) factors were calculated for each thermoluminescent dosimeter (TLD), and the TLDs which are within $\pm 5 \%$ acceptance limit were selected for use. In total, 116 lithium fluoride TLDs (TLD-100 LiF) were used for each measurement. The TLDs used are in the form of a square prism with dimensions $3.2 \mathrm{~mm} \times 3.2$ $\mathrm{mm} \times 0.9 \mathrm{~mm}$. In order to make the measurements more accurate, each TLD has been calibrated individually before measurements for the photon energy of $100 \mathrm{kVp}$ and $120 \mathrm{kVp}$. Same beam parameters were used for the calibration as during the measurements (Fig. 4). TLDs were irradiated in a poly-methyl methacrylate holder. After that, irradiated TLDs were read with Harshaw 3500 TLD reader (Harshaw Thermo Electron, Solon, USA). From the beam and dose parameters used in the calibration process (mAs, mGy), Reader Calibration Factor (RCF) was computed for each scanning protocol which was used in the calculation procedure of patient organ doses.

\section{Calculation of organ doses and effective dose}

Patient organ dose measurements were carried out in an Alderson Rando anthropomorphic male phantom by TLD placed in hole positions of organs. The definition of equivalent dose is based on the mean absorbed dose, $D_{T}$, in the volume of a specified organ or tissue $\mathrm{T}$, due to radiation of type $\mathrm{R}$ [12]. The unit of absorbed dose is Gray (Gy). The mean absorbed dose, $\mathrm{D}_{\mathrm{T}}$ to a specific organ or tissue $\mathrm{T}$, is calculated by using equation 1 [12]. 


$$
D_{T}=\sum_{i} D_{i} f_{i}
$$

where $\mathrm{D}_{\mathrm{T}}$ denotes the mean absorbed dose of the specified organ in miligray ( $m G y), D_{i}$ is the arithmetic mean dose of the TLDs for the corresponding organ within the slice $i$ of phantom, $f_{\mathrm{i}}$ denotes the fraction of total organ mass in the slice $i$ of the Alderson RANDO phantom. Equivalent dose in an organ or tissue, $\mathrm{H}_{\mathrm{T}}$, is then defined by equation 2 [12]:

$$
H_{T}=\sum_{R} w_{R} D_{T}
$$

where $w_{R}$ is the radiation weighting factor for radiation $\mathrm{R}$. Radiation weighting factor is equal to one for photons [12].

After calculating patient equivalent organ doses $\left(H_{T}\right)$, effective dose $\left(D_{\text {eff }}\right)$ were computed in milisievert $(\mathrm{mSv})$ by using Equation 3 which is defined by the International Commission on Radiological Protection (ICRP). The effective dose, $\mathrm{D}_{\text {eff }}$, is defined by a weighted sum of organ or tissue equivalent doses [12].

$$
D_{e f f}=\Sigma_{T} w_{T} H_{T}
$$

where $w_{T}$ is the tissue weighting factor prescribed by ICRP [12].

In this study, the tissue weighting factors, which were suggested in the ICRP 103 publication, were used [12]. In addition to that, 0.0086 value was assigned for remainder organs as suggested by the ICRP 103 publication. Besides, while in the ICRP 60 report, the upper large intestine (ULI) was included in remainder organs, in the ICRP 103 report, the upper large intestine and lower large intestine (LLI) were combined and named as colon. Moreover, in this study, while calculating the colon dose, the formula suggested in the ICRP 67 report was used, which is mass weighted average of the doses of both ULI and LLI as Colon Dose $=0.57$ $U L I+0.43$ LLI [12-14].

Fraction values of the skin were obtained from the study of Huda \& Sandison [15]. All the other organs fractions were reported in literature. Because of the lack of information about the hole location for red bone marrow (RBM) and bone surface in the RANDO phantom, the equivalent doses were not able to be measured for these organs. In addition to that, since an Alderson RANDO male phan- tom was used, the breast dose was not measured. Moreover, because of the gonads were out of the beam line in pelvic irradiation, the equivalent dose of the gonads was not calculated. As a result, the effective dose was underestimated for the patient.

\section{Results}

The maximum organ dose for $\mathrm{H} \& \mathrm{~N}$, thoracic and pelvic scans were at the lens, heart and small intestine, with equivalent doses value of $3.43 \mathrm{mGy}$, $22.94 \mathrm{mGy}$ and $25.28 \mathrm{mGy}$, respectively. The calculated effective dose to the patient for H\&N S20, Chest M20 and Pelvis M20 were $0.25 \mathrm{mSv}, 5.56 \mathrm{mSv}$ and $4.72 \mathrm{mSv}$, respectively. The measured organ doses and calculated effective dose to the patient are displayed in Table 2.

\section{Discussion}

Position accuracy, which is among the most determining factors that affect the quality of treatment, is performed in a flawless manner with devices using advanced technology such as $\mathrm{CBCT}$. Because of that, CBCT is of critical importance in today's radiotherapy treatment, and researchers have examined the quality assurance parameters of this system from the beginning of implementation [16-21].

On the other hand, beside this unique properties of CBCT, delivering to the patient an additional dose for this procedure is its major drawback. In this study, the absorbed organ doses and the effective dose given to the patient were measured on an Alderson RANDO phantom for the imaging protocols applied to different parts of the body using the $\mathrm{kV}$ CBCT imaging system on the Elekta Synergy Platform.

The organ doses highly rely on exposure parameters and the geometry of the scanning procedure, such as field of view size and depth of measurement. In literature, a large spectrum of research has been conducted. Although most of the studies were carried out with similar exposure parameters, a considerable number of them applied different scanning parameters.

Most of the recent studies have indicated that secondary cancer risk due to X-ray exposure from diagnostic imaging of the CBCT systems is more than anticipated, especially for pediatric patients [7]. 
In this study, the calculated doses were just for a single course of an imaging process. Because of that, treatment delivery dose of radiation therapy may differ for different types of cancer and the total imaging dose for the entire course of radiotherapy might be increased. In other words, for a single patient, the obtained values in Table 2 could be multiplied by the total fraction number of the radiotherapy. In such case, the dose taken from the imaging process could rise to higher values during the course of treatment.

Hyer et al. measured effective dose with optical-fiber based scintillation detectors for the Elekta Synergy XVI system. It was one of the most comprehensive study in literature for investigating the organ doses from CBCT imaging. Although similar exposure parameters for $\mathrm{H} \& \mathrm{~N}$ and Chest $\mathrm{kV} C B C \mathrm{C}$ irradiations were used; our results in this study are different. In the chest scanning protocol, the organs doses, such as the heart, lung, liver, stomach and kidney, were calculated to be lower. In addition, while the dose to the esophagus was found to be close to the measured value in this study; the thyroid dose was calculated higher as $19.24 \mathrm{mGy}$. On the other hand, all measured organ doses for $\mathrm{H} \& \mathrm{~N}$ scanning protocol were lower than that of those calculated in this study. Furthermore, the effective dose measurements reported for $\mathrm{H} \& \mathrm{~N}$ and Chest irradiation were consistent with the findings of our study which were $0.04 \mathrm{mSv}$ and $7.15 \mathrm{mSv}$, respectively [9].

Remarkably, although higher exposure parameters were applied for $\mathrm{H} \& \mathrm{~N}$ imaging in the study of Amer et al., the obtained surface dose of the phantom was consistent with our study [22].

A feasibility study was carried out for the Elekta Synergy XVI system on the Alderson rando phantom by Sykes et al. In that study, the image quality and the delivered dose values were compared in high exposure and low exposure scanning modes. In $\mathrm{H} \& \mathrm{~N}$ imaging, it was reported that surface and internal absorbed dose values were measured to be about $1 \mathrm{mGy}$, which was performed with the same exposure parameters in this study. The resultant values were approximately 1 in 3 of the values obtained in the present study [23].

Moon et al. used glass dosimeters to measure patient organ doses in a RANDO female phantom with the same exposure parameters and scanning geometry for the chest and pelvic scan of the cur- rent study. The reported organ doses were in good agreement with those obtained from this study for the chest scan, particularly for the lungs, stomach, liver and thymus. On the other hand, the doses measured from the pelvic scan were lower for almost all specified organs. Nevertheless, the effective dose value for the pelvic scan was completely consistent with this study, which was $4.09 \mathrm{mSv}$. The underlying reason for that consequence can be that the number of tissues included in the calculation was higher than that of our study [24].

Dufek et al. evaluated and compared the organ and effective doses for different version of OBI and XVI systems in an Alderson RANDO male phantom by using a great number of TLDs. Although the exposure parameters for $\mathrm{H} \& \mathrm{~N}$ scans were similar, the calculated organ doses were lower than this study. The doses for most of the organs in the H\&N region were lower than $0.1 \mathrm{mGy}$ in that study. The maximum organ doses reported for the salivary gland and oral mucosa as $0.9 \mathrm{mGy}$ and $1.1 \mathrm{mGy}$, respectively. These measured doses were lower than the doses obtained in this study, i.e. about $3 \mathrm{mGy}$ [25].

In this study, similar to the studies in the literature, it is shown that decreasing dose parameter values, particularly $\mathrm{mAs}$ and the projection numbers, are critical factors in the reduction of the dose delivered to the patient.

It should be noted that during the imaging process, trading off image quality in order to give the patient lower dose may cause repeat imaging. In other words, it is important to be able to achieve the "As low as diagnostically acceptable" (ALADA) principle without ignoring the "As low as reasonably achievable" (ALARA) principle. This understanding will allow both the reduction of the dose given to the patient and an imaging procedure with sufficient imaging quality.

\section{Conclusions}

In this study, the equivalent doses of 20 organs and effective dose to patients were measured with TLDs in an Alderson RANDO male phantom for Elekta Synergy XVI system with the most used clinical protocols of $\mathrm{kV} \mathrm{CBCT}$. The equivalent organ doses and effective doses were estimated according to the ICRP 103 recommendations. The obtained findings were consistent with most of the published 
results in literature. Although the doses to patient organs from the $\mathrm{kV}$ CBCT system were relatively low when compared with the prescribed treatment dose, the amount of delivered dose should be monitored and recorded carefully in order to avoid secondary cancer risk, especially in pediatric examinations.

\section{Conflict of interest}

None declared.

\section{Funding}

This research did not receive any specific grant from funding agencies in the public, commercial, or not-for-profit sectors. None of the authors have any financial support or relationships that may pose conflict of interest.

\section{Acknowledgements}

This research did not receive any specific grant from funding agencies in the public, commercial, or not-for-profit sectors. None of the authors have any financial support or relationships that may pose conflict of interest.

\section{References}

1. Stewart BW, Wild C. 2014 World Cancer Report . International Agency for Research on Cancer, Lyon 2014.

2. Xu XG, Bednarz B, Paganetti H. A review of dosimetry studies on external-beam radiation treatment with respect to second cancer induction. Phys Med Biol. 2008; 53(13): R193-R241, doi: 10.1088/0031-9155/53/13/R01, indexed in Pubmed: 18540047.

3. Jaffray DA. Emergent technologies for 3-dimensional image-guided radiation delivery. Semin Radiat Oncol. 2005; 15(3): 208-216, doi: 10.1016/j.semradonc.2005.01.003, indexed in Pubmed: 15983946.

4. Akyalcin S, English JD, Abramovitch KM, et al. Measurement of skin dose from cone-beam computed tomography imaging. Head Face Med. 2013; 9: 28, doi: 10.1186/1746160X-9-28, indexed in Pubmed: 24192155.

5. Ernst $M$, Manser $P$, Dula K, et al. TLD measurements and Monte Carlo calculations of head and neck organ and effective doses for cone beam computed tomography using 3D Accuitomo 170. Dentomaxillofac Radiol. 2017; 46(7): 20170047, doi: 10.1259/dmfr.20170047, indexed in Pubmed: 28749697.

6. Boyer AL, Antonuk L, Fenster A, et al. A review of electronic portal imaging devices (EPIDs). Med Phys. 1992; 19(1): 1-16, doi: 10.1118/1.596878, indexed in Pubmed: 1620036.

7. Dzierma Y, Mikulla K, Richter P, et al. Imaging dose and secondary cancer risk in image-guided radiotherapy of pediatric patients. Radiat Oncol. 2018; 13(1): 168, doi: 10.1186/ s13014-018-1109-8, indexed in Pubmed: 30185206.
8. Hess $\mathrm{CB}$, Thompson HM, Benedict $\mathrm{SH}$, et al. Exposure Risks Among Children Undergoing Radiation Therapy: Considerations in the Era of Image Guided Radiation Therapy. Int J Radiat Oncol Biol Phys. 2016; 94(5): 978-992, doi: 10.1016/j.ijrobp.2015.12.372, indexed in Pubmed: 27026304.

9. Hyer DE, Serago CF, Kim S, et al. An organ and effective dose study of XVI and OBI cone-beam CT systems. J Appl Clin Med Phys. 2010; 11(2): 3183, doi: 10.1120/jacmp. v11i2.3183, indexed in Pubmed: 20592702.

10. GolikovVY, Nikitin VV. Estimation of the mean organ doses and the effective dose equivalent from Rando phantom measurements. Health Phys. 1989; 56(1): 111-115, indexed in Pubmed: 2909497.

11. Scalzetti EM, Huda W, Bhatt $S$, et al. A method to obtain mean organ doses in a RANDO phantom. Health Phys. 2008; 95(2): 241-244, doi: 10.1097/01. HP.0000310997.09116.e3, indexed in Pubmed: 18617806.

12. International Commission on Radiological Protection (ICRP). The 2007 Recommendations of the International Commission on Radiological Protection. ICRP Publication 103. Ann ICRP. 2007; 37(2-4): 1-332.

13. International Commission on Radiological Protection.. 1991Recommendations of the International Commission of Radiological Protection. ICRP Publication 60. Pergamon Press, Oxford 1991.

14. International Commission on Radiological Protection. Age-dependent Doses to Members of the Public from Intake of Radionuclides - Part 2 Ingestion Dose Coefficients. ICRP Publication 67. Ann. ICRP. 1997; 23(3-4).

15. Huda W, Sandison GA. Estimation of mean organ doses in diagnostic radiology from Rando phantom measurements. Health Phys. 1984; 47(3): 463-467, indexed in Pubmed: 6500950.

16. Dzierma $Y$, Ames E, Nuesken F, et al. Image quality and dose distributions of three linac-based imaging modalities. Strahlenther Onkol. 2015; 191(4): 365-374, doi: 10.1007/ s00066-014-0798-7, indexed in Pubmed: 25527311.

17. Marchant TE, Joshi KD, Moore CJ. Accuracy of radiotherapy dose calculations based on cone-beam $\mathrm{CT}$ : comparison of deformable registration and image correction based methods. Phys Med Biol. 2018; 63(6): 065003, doi: 10.1088/1361-6560/aab0f0, indexed in Pubmed: 29461255.

18. Conrad M, Bolard G, Nowak M, et al. Determination of the effective dose delivered by image guided radiotherapy in head \& neck and breast treatments. Z Med Phys. 2018; 28(4): 276-285, doi: 10.1016/j.zemedi.2018.01.001, indexed in Pubmed: 29426589.

19. Ding GX, Munro P. Radiation exposure to patients from image guidance procedures and techniques to reduce the imaging dose. Radiother Oncol. 2013; 108(1): 91-98, doi: 10.1016/j.radonc.2013.05.034, indexed in Pubmed: 23830468.

20. Ariyaratne $\mathrm{H}$, Chesham $\mathrm{H}$, Pettingell J, et al. Image-guided radiotherapy for prostate cancer with cone beam $\mathrm{CT}$ : dosimetric effects of imaging frequency and PTV margin. Radiother Oncol. 2016; 121(1): 103-108, doi: 10.1016/j. radonc.2016.07.018, indexed in Pubmed: 27576431.

21. Son K, Kim JS, Lee H, et al. Imaging dose of human organs from kV-CBCT in image-guided radiation therapy. Radiat Prot Dosimetry. 2017; 175(2): 194-200, doi: 10.1093/rpd/ ncw285, indexed in Pubmed: 27765832. 
22. Amer A, Marchant T, Sykes J, et al. Imaging doses from the Elekta Synergy X-ray cone beam CT system. Br J Radiol. 2007; 80(954): 476-482, doi: 10.1259/bjr/80446730, indexed in Pubmed: 17684077.

23. Sykes JR, Amer A, Czajka J, et al. A feasibility study for image guided radiotherapy using low dose, high speed, cone beam X-ray volumetric imaging. Radiother Oncol. 2005; 77(1): 45-52, doi: 10.1016/j.radonc.2005.05.005, indexed in Pubmed: 16157400.
24. Moon Y, Kim HJ, Kwak D, et al. Effective dose measurement for cone beam computed tomography using glass dosimeter. Nucl Engineer Tech. 2014; 46(2): 255-262, doi: 10.5516/net.08.2012.080.

25. Dufek V, Horakova I, Novak L. Organ and effective doses from verification techniques in image-guided radiotherapy. Radiat Prot Dosimetry. 2011; 147(1-2): 277-280, doi: 10.1093/rpd/ncr335, indexed in Pubmed: 21816726. 